\title{
Impact of the Spanish smoking legislation among smokers: A longitudinal study with biomarkers in Barcelona (Spain)
}

Cristina Lidón-Moyano, $\mathrm{BSc}, \mathrm{MSc}^{1}$; Marcela Fu, BSc, $\mathrm{PhD}^{2,3,4}$; Raúl Pérez-Ortuño, $\mathrm{PhD}^{7}$; Montse Ballbè, MSc, $\mathrm{PhD}^{2,3,4,5,6}$; Ariadna Feliu, $\mathrm{MPH}^{2,3,4}$; Juan Carlos Martín-Sánchez, BSc, PhD ${ }^{1}$; Nuria Matilla-Santander, $\mathrm{MPH}^{1}$; José A. Pascual, $\mathrm{PhD}^{7,8}$; Esteve Fernández, MD, $\mathrm{PhD}^{2,3,4,5}$; and Jose M. Martínez-Sánchez, MPH, $\mathrm{PhD}^{1,2,3^{*}}$

${ }^{1}$ Group of Evaluation of Health Determinants and Health Policies, Universitat Internacional de Catalunya, Sant Cugat del Vallès, Spain

${ }^{2}$ Tobacco Control Unit, Cancer Prevention and Control Program, Institut Català d'Oncologia, L'Hospitalet de Llobregat, Barcelona, Spain

${ }^{3}$ Cancer Prevention and Control Group, Institut d'Investigació Biomèdica de Bellvitge - IDIBELL, L'Hospitalet de Llobregat, Barcelona, Spain

${ }^{4}$ Department of Clinical Sciences, School of Medicine, Universitat de Barcelona, Barcelona, Spain

${ }^{5}$ Catalan Network of Smoke-free Hospitals, L'Hospitalet de Llobregat, Barcelona, Spain

${ }^{6}$ Addictions Unit, Institute of Neurosciences, Hospital Clínic de Barcelona,Barcelona, Spain

${ }^{7}$ Group of Integrative Pharmacology and Systems Neuroscience, Neurosciences Programme, IMIM (Hospital del Mar Medical Research Institute), Parc de Recerca Biomèdica de Barcelona, Barcelona, Spain

${ }^{8}$ Department of Experimental and Health Sciences, Universitat Pompeu Fabra, Parc de Recerca Biomèdica de Barcelona, Barcelona, Spain

\section{*Corresponding author:}

Jose M. Martínez Sánchez, BSc, MPH, PhD

Group of Evaluation of Health Determinants and Health Policies

Departament de Ciències Bàsiques

Universitat Internacional de Catalunya

Carrer de Josep Trueta s/n

08195 Sant Cugat del Vallès (Barcelona)

TLF: 935042018

E-mail: jmmartinez@uic.es

Word count: 2,539 


\section{ABSTRACT}

Background: The aim of this study is to assess the impact of the Spanish tobacco control legislation on smoking behavior and salivary cotinine concentration among smokers.

Methods: We used data from a longitudinal study, before (2004-2005) and after (2013-2014) the implementation of the two national smoking bans (in 2006 and 2011), in a representative sample of adults ( $\geq 16$ years old) from Barcelona (Spain). We only analyzed a subsample of continuing smokers ( $n=116$ ). We conducted a survey on smoking behavior and obtained saliva sample for cotinine analyses. We calculated geometric means (GM).

Results: The salivary cotinine concentration significantly increased $28.7 \%$ (GM from $91.7 \mathrm{ng} / \mathrm{ml}$ to $117.3 \mathrm{ng} / \mathrm{ml}, \mathrm{p}=0.015)$ after the implementation of the two Spanish smoke-free bans. Nonetheless, no pattern of change was observed in the self-reported number of cigarettes smoked daily.

Conclusions: Our study shows a significant increase in the salivary cotinine concentration among adult continuing smokers after both Spanish legislations. This increase could be due to differences in smoking topography (increase in the depth of inhalation when smoking) along with changes in the type of tobacco smoked (increase in smoking roll your own cigarettes or mixed used). Our results suggest the need to extend tobacco control policies, focusing on reduction of use of any kind of tobacco product and implementing better treatment policies to help smokers stop smoking.

Keywords: Smoke-free legislation; Smoking; Cotinine; Biomarker; Tobacco consumption; FTCD; 


\section{INTRODUCTION}

Tobacco is the first single leading cause of preventable death in the world (1), being related to more than 25 diseases and being responsible for $30 \%$ of all cancers, respiratory diseases and cardiovascular diseases (2). In 2015, WHO estimated that about 15\% (1.1 billion people) of worldwide population smoked (3) and that tobacco use is responsible for about six million deaths across the world each year, including about 600,000 deaths from the effects of secondhand smoke (SHS) (4). In Spain, the latest data reported showed that $28 \%$ of men and $19 \%$ of women were tobacco users in 2014 (5) and that more than 60,000 deaths per year were attributable to active smoking (6).

Consequently, two smoke-free laws have been passed in Spain after the approval of the World Health Organization Framework Convention on Tobacco Control (WHO FCTC) (7). On the $1^{\text {st }}$ of January, 2006, a smoke-free legislation came into effect (Law 28/2005). The law was a compendium of public health measures against smoking and included regulations on publicity, sale, supply, and consumption of tobacco products (8). Smoking was banned in all indoor workplaces, public places, public transport facilities including enclosed stations, hospitals and other health care facilities, schools and universities as well as in retail stores and shopping centres. However, hospitality venues were subject to only a partial ban. In bars and restaurants smaller than $100 \mathrm{~m} 2$, the proprietor could choose between permitting or prohibiting smoking. Those larger than $100 \mathrm{~m} 2$ were defined as smoke-free, but the law allowed the proprietor to provide a physically separated and independently ventilated smoking area comprising less than $30 \%$ of the total floor area. Moreover, several tax reforms followed the implementation of the partial ban (9). This law was a great advance for public health in Spain; however, it was not complete in terms of health protection from SHS exposure. The scientific evaluation of this law showed the need to promote a total ban (10-12). On the $2^{\text {nd }}$ of January, 2011, a new law came into force (Law 42/2010), including several tax reforms and 
extending the smoke-free regulation to all hospitality venues without exception (9) and to some outdoor areas, including hospital premises, educational campuses, and playgrounds. However, designated smoking rooms are still permitted in psychiatric services, nursing homes, prisons and up to $30 \%$ of hotel rooms.

The principal aim of both Spanish tobacco control legislations was to protect non-smoking population from tobacco smoke exposure. Therefore, their scientific evaluation focused on their effect on SHS reduction, often neglecting research about the effect on active smokers. In this sense, a systematic review (13) has concluded that the implementation of smoke-free policies and restrictions in public spaces, workplaces or residences lead to a decrease in smoking prevalence and cigarette consumption. Moreover, previous studies $(14,15)$ suggest that active smokers could be changing their smoking pattern to consuming cheaper tobacco products, such as Roll Your Own (RYO) cigarettes, which are subject of little taxation in Spain (16). In addition, RYO are usually not filtered (17) and as a result more nicotine (as well as other toxicants) is delivered with smoke. There are few studies that assess the impact of smoking legislation among the smoking population using a specific biomarker of tobacco smoke absorption such as cotinine, which is the major proximate metabolite of nicotine (18). In Spain, a previous study assessed the impact of the first legislation among hospitality workers who smoke, using self-reported data and information regarding salivary cotinine (19). Cotinine concentration in biological fluids (blood, urine or oral fluid, widely referred to as saliva) (20) indicates tobacco exposure over the previous 1-2 days (21) and is strongly correlated with the number of cigarettes smoked daily (22). Moreover, the cotinine concentration could vary within each stage of change (defined in the methods section), according to the number of cigarettes smoked, time to first cigarette of the day, and nicotine dependence (23).

Therefore, the objective of this study is to assess the impact of the Spanish tobacco control legislations (laws 28/2005 and 42/2010) on smoking behavior and salivary cotinine 
concentration among smokers by using a cohort study of adult smokers in Barcelona (Spain) along with biomarker information (salivary cotinine concentration).

\section{METHODS}

This is a longitudinal study (cohort study) from a representative sample of the adult population ( $\geq 16$ years at baseline, $\geq 25$ at follow-up) of the city of Barcelona (Catalonia, Spain). The baseline study was carried out during the years 2004-2005 $(24,25)(n=1,245)$ and the followup took place in 2013-2014, after the implementation of both Spanish tobacco control legislations.

To obtain the baseline sample, a representative random sample by age, sex, and district was drawn from the official 2001 population census of Barcelona, a reliable source of populationbased information explained elsewhere $(24,25)$. Briefly, the procedure was as follows: a personal letter was sent to eligible participants, and trained interviewers contacted the subjects at home and informed them about the study. When the index person was not contacted (after several attempts following a strict protocol that included visits on weekends and during non-working hours) or refused to participate, we randomly selected a substitute in the same sex-, age-, and district-group. We asked participants to answer a face-to-face questionnaire and to sign a consent form in order to be contacted in the follow-up. From the baseline sample, we excluded 235 subjects: 150 after checking their data in the Insured Central Registry of Catalonia (101 died and 49 migrated out of the province of Barcelona) and 85 subjects who did not give consent to be followed up or were minors ( $<18$ years old) in 20042005 and we did not ask consent to re-contact. The percentage of follow-up in this first stage was $81.1 \%$. The follow-up was conducted in $2013-2014$. In total, $72.9 \%$ of the eligible sample agreed to participate (736 out of 1010 traced, second stage of follow-up), $18.5 \%$ refused to participate, $7.2 \%$ had moved elsewhere and $1.4 \%$ had died. The final sample included 736 individuals (Figure 1) and was skewed as slightly older in comparison with the general 
population of Barcelona. For this reason, we weighted our data according to the age distribution of the city of Barcelona to maintain its representativeness. The percentage of participation in both stages was $51.9 \%$ (736 out 1245 ). We did not find statistically significant differences between the followed-up sample $(n=736)$ and the participants lost in the second stage $(n=274)$ according to age, sex, educational level (categorized as low: unschooled, elementary school completed or uncompleted and special education; intermediate: high school and training cycles; and high: university education) and smoking status. However, we described statistically significant differences according to age, level of education and smoking status between the follow-up sample $(n=736)$ and the participants lost in both stages of the follow-up ( $n=509)$ (Table 1).

We asked the participants to provide a $9 \mathrm{ml}$ sample of saliva for cotinine analysis, using the same protocol before and after the Spanish tobacco control legislations. Participants were asked to rinse their mouths and then suck a lemon candy (Smint) to stimulate saliva production. Saliva samples were frozen and sent to the IMIM-Hospital del Mar Medical Research Institute in Barcelona. Saliva samples were analyzed using liquid chromatographytandem mass spectrometry (LC/MS/MS) with multiple reaction monitoring. The limit of quantification was $0.4 \mathrm{ng} / \mathrm{ml}$ (quantification error was <15\%) (26).

We used the same core questionnaire in both surveys to gather relevant information on smoking characteristics, along with the information on their cotinine concentration. Smoking status, was obtained from the question: "Which of the following statements better describes your smoking status?" with the possible answers: 'Nowadays I smoke everyday (at least one cigarette per day)', these are current daily smokers; 'Nowadays I smoke occasionally (less than one cigarette per day)', these are current occasionally smokers; 'I don't smoke now, but I smoked before every day', these are former daily smokers; 'I don't smoke now, but I smoked before occasionally', these are former occasionally smokers; and 'I have never smoked', these 
are never smokers. We also aggregated these categories when appropriate as 'smokers' (current daily and occasionally smokers) and 'non-smokers' (former daily and occasionally smokers plus never-smokers). The percentage of self-reported smokers with salivary cotinine inconsistent with active smoking ( $\leq 35 \mathrm{ng} / \mathrm{ml}$ per cigarette smoked daily (27)) was $3.4 \%$ before the legislations and $12.1 \%$ after the legislations, accounting for $13.8 \%$ of the total sample of continuing smokers ( $n=116)$. Moreover, when using the self-reported number of cigarettes smoked in the last 24 hours and in the last 48 hours, the percentage of inconsistent smokers in the total sample of continuing smokers decreased to $6.0 \%$ and $0.8 \%$ respectively. However, for this analysis, we used self-reported smoking status for the identification of current smokers in order to maximize the available sample size. The final sample for this analysis consists of 116 continuing smokers, that is to say, those who self-reported active smoking before and after the legislation (Figure 1).

From the same questionnaire before and after the two laws, we also obtained information about the type of tobacco smoked, obtained through the question: "What kind of tobacco product do you habitually consume?" with the possible answers: "cigarettes", "RYO cigarettes", "cigars", "little cigars", "pipes", "hookah" and "e-cigarettes". The answers to this question were dichotomized as 'CC-CC' indicating those who only smoked conventional cigarettes (CC) before and after the two laws, 'CC - RYO', indicating those who switched from conventional to RYO cigarettes, 'CC $-\mathrm{MU}$ ', indicating those who switched from conventional to mixed use (CC and RYO) and 'Other', indicating other possible options. Moreover, we collected self-reported information about the smokers' stage of change (28): precontemplation, when smokers were not seriously considering quitting within the next 6 months; contemplation, when smokers were seriously considering quitting within the next 6 months but not within the next 30 days; and preparation, when smokers were planning to quit within the next 30 days and had attempted to quit for at least 24 hours in the past year. The information to obtain 
smoker's stage of change was obtained through two different questions. 1) "Are you seriously considering the possibility of...?" with the possible answers: "quit smoking during the following week', 'quit smoking during the following month', 'quit smoking during the following three months', quit smoking during the following six months', 'quit smoking during the following twelve months', 'quit smoking but not during the following twelve months', and 'no quit smoking'. 2) "In the last year, have you been at least one day without smoking?" (not taking into account days in which you have been ill), with the possible answers: 'yes', 'no', and 'doesn't know or doesn't answer'. We also gathered information about the number of cigarettes smoked daily (either conventional or RYO cigarettes) and the Fagerström Test for Cigarette Dependence (FTCD) score (28) categorized as 'low' ( $\leq 4)$, 'medium' (5) and 'high' ( $\geq 6$ ). FTCD score includes six items: time to first cigarette $(0-3$ points $)$, difficulty to refrain $(0-1$ points), hardest cigarette to give up ( $0-1$ points), cigarettes per day ( $0-3$ points), smoking more in the morning than in the rest of the day $(0-1$ points), and smoking while ill $(0-1$ points). FTCD score ranges from 0 to 10 and assesses distinguishable self-reportable pharmacological dimensions of nicotine addiction (29). Finally, we gathered self-reported information about several smoking characteristics, such as use of regular or non-regular cigarettes (light, ultralight, etc.), type of tobacco smoked (blond or black), use of cigarettes with or without filter, length of cigarettes left after smoking (in centimeters), depth (superficial, intermediate, deep) and frequency of inhalation (continuous, regular, scarce).

For statistical analysis we calculated geometric means (GM) and their geometric standard deviation (GSD), given the skewed distribution of cotinine concentration. We used linear mixed effect models with individuals as random effects to model the change in cotinine concentration (after log10 transformation), their 95\% confidence intervals and the p-value, adjusted for sex, age, and educational level. The results were stratified by sex, age, educational level, kind of tobacco smoked, FTCD score and stages of change. We also used generalized 
linear mixed models with individuals as random effects to calculate the prevalence ratio of the change in smoking characteristics. The statistical programs used were R-3.0.2 and Stata v14.

\section{RESULTS}

When analysing self-reported data from the whole sample $(n=736), 166$ participants $(22.6 \%$, 95\% Cl: 19.7-25.8) were continuing smokers and 456 participants (62.0\%, 95\% Cl: 58.4-65.5) were continuing non-smokers (never and former smokers). In other words, they showed the same smoking status at baseline and at follow-up. Moreover, 88 participants (11.9\%) quit at follow-up and 26 participants (3.5\%) initiated or relapsed tobacco use at the follow up (2 participants, $0.2 \%$, were never smokers at baseline and 24 participants, $3.3 \%$, were former smokers).

According to cotinine levels from the whole sample ( $n=503$ due to missing data), 115 participants $(22.9 \%, 95 \% \mathrm{Cl}: 19.3-26.8)$ were continuing smokers and 360 participants $(71.6 \%$, 95\% Cl: 67.4-75.4) were continuing non-smokers (never and former smokers). Moreover, 20 participants (4.0\%) quit at follow-up, 5 participants (1.0\%) initiated or relapsed tobacco use at the follow up, and 3 participants $(0.6 \%)$ were not properly classified.

In addition, when comparing self-reported smoking status with cotinine classification at baseline ( $n=566$ due to missing data), 144 out of 207 (69.6\%) self-reported smokers were correctly classified as such according to cotinine levels, and $100 \%$ of self-reported non-smokers (359) were also correctly classified. Similarly, when comparing at the follow-up ( $n=582$ due to missing data), 107 out of 140 (76.4\%) self-reported smokers were correctly classified as such according to cotinine levels, and 428 out of 442 (96.8\%) of self-reported non-smokers were also correctly classified. 
Among self-reported continuing smokers, we observed a general increase in salivary cotinine concentration after the implementation of the two Spanish smoke-free laws (Figure 2, panel B). Salivary cotinine concentration significantly increased by $28.7 \%$ (GM from $91.1 \mathrm{ng} / \mathrm{ml}$ to $117.3 \mathrm{ng} / \mathrm{ml}, \mathrm{p}=0.015$ ) after the implementation of the two Spanish smoke-free laws (Table 2). The increase in the salivary concentration was statistically significant among young people, highly educated and among those who switched from conventional to RYO (Table 2). In addition, a statistically significant decrease was found in the proportion of individuals classified in the medium category of FTCD score (from $23.3 \%$ to $9.1 \%, p=0.017$ ) when comparing pre and post legislations (Figure 2, panel A). Nonetheless, no clear pattern of change was observed in the number of cigarettes smoked daily (Figure 2, panel C). Even though, a non-significant increase in the number of cigarettes smoked daily can be observed among those who switched from conventional cigarettes only to mixed use (from 16.6 cigarettes/day to 24.3 cigarettes/day, $p=0.270$ ) when differentiating between type of tobacco smoked (Figure 2, panel C). In this regard, $8.3 \%$ of smokers switched from conventional to mixed use, $13.0 \%$ switched from conventional to RYO, and $59.1 \%$ smoked only conventional cigarettes before and after the two laws.

Regarding other smoking characteristics (Table 3), we observed an increase in the use of regular cigarettes (from $66.6 \%$ to $76.3 \%, p=0.019$ ), in the length of cigarettes left after smoking (from $1.13 \mathrm{~cm}$ to $2.26 \mathrm{~cm}, \mathrm{p}=0.023$ ) and in the depth of inhalations when smoking at the expense of the reduction of intermediate depth of inhalation (deep inhalation from $42.4 \%$ to $57.5 \%, p=0.001$ ) along with a reduction in low frequency of inhalation (from $28.2 \%$ to $10.1 \%$, $\mathrm{p}=0.036)($ Table 3$)$.

\section{DISCUSSION}


Among continuing smokers we found an increase in salivary cotinine concentration of around $29 \%$ after both Spanish tobacco control legislations, particularly among younger and highly educated smokers. We also observed a switch in the type of tobacco used, from conventional to RYO cigarettes or to mixed use in $21.3 \%$ of smokers. In addition, we found a statistically significant decrease in the proportion of individuals classified as medium in the FTCD score, which represents those individuals with a FTCD score of 5 out of 10, that is to say, medium dependence to nicotine.

The hardening hypothesis (30) suggests that smokers who quit over the time are those who are less dependent, and the remaining smokers are more dependent. However, previous studies have counteracted this hypothesis (31-33) using self-reported questionnaires to measure the tobacco or nicotine dependence. In this sense, we did not find differences in salivary cotinine concentration, nor in FTCD scores, among those who quit smoking after Spanish tobacco control legislations and those who continue smoking (data not shown). On the other hand, we found an increase in salivary cotinine among continuing smokers. In this sense, when considering biomarkers as a proxy of tobacco dependence (34), our results could indicate that continuing smokers became more dependent after Spanish tobacco control legislations. However, our study did not show any significant increase in the FTCD score among smokers who continue smoking; this could be due to the fact that FTCD only measures the dependence of conventional manufactured cigarettes (29). A previous study showed that the FTCD has some limitations, such as low internal consistency, floor effects, and that it does not address important aspects of addiction to cigarettes (35). Although there is a positive relation between the FTCD score, tobacco consumption and salivary cotinine $(34,35)$, other factors might also have an effect on cotinine concentration, such as diet and meals, age, sex, race, pregnancy, kidney disease, use of estrogen-containing hormone preparations and other medications (36). In our work, the effect of age may play a prominent role given the 
longitudinal structure of our data, that is to say, given that the cohort has aged by ten years between baseline and follow-up. In this sense, a previous review showed that total clearance of nicotine lowered by $23 \%$ in the elderly (age $>65$ ) compared to adults (37). If nicotine is cleared at a slower rate, it will lead to a prolonged exposure (i.e. it would take more time to remove cotinine from the system), and it could also yield to obtain higher levels of cotinine under the same smoking consumption patterns. Therefore, the observed increase in salivary cotinine could be related to the aged of our cohort. However, the increase in the older group of age at follow-up was only of $11 \%$, which represents 13 individuals out of the 116 continuing smokers. Our models were adjusted by sex, age and educational level, obtaining similar results in the adjusted and unadjusted models. Moreover, smoking topography (24) could also affect cotinine concentration.

According to our results, an increase in smoking regular cigarettes, in the centimeters left without smoking and in the depth of inhalation when smoking, along with a reduction in low frequency of inhalation, can be observed after the application of the Spanish tobacco laws. This could be explained because, under these smoking restriction policies, smokers have fewer opportunities to smoke in public places and less time to do so. Thus, changes in the smoking topography may be evident. In addition, the low increase in salivary cotinine found in our study in high dependent smokers at baseline could be due to a ceiling effect, since the highest value of salivary cotinine before bans was observed in this group. Furthermore, it is important to bear in mind that the information about FTCD score and the number of cigarettes smoked daily was self-reported, therefore it can be subject to potential limitations related to survey based studies. Further research is needed to analyze possible factors related to cotinine concentration, as well as to ensure cotinine concentration properly measures nicotine dependence. 
The increase in the salivary concentration was statistically significant among young people, highly educated, and among those who switched from conventional cigarettes to RYO. These results are likely to be related, since previous works (38) showed a great increase in the consumption of hand-rolled tobacco and other tobacco products, especially among young people; in fact, according to those results, older people did not smoke hand-rolled tobacco, neither before nor after the implementation of the Spanish smoke-free legislations. Moreover, we found a significant association between age and education, age and type of tobacco smoked, and education and type of tobacco smoked (data not shown). Therefore, the increase in the salivary concentration among high-educated individuals might be confounded by age.

Additionally, our study showed a switch from conventional to RYO cigarettes or to mixed use in $21.3 \%$ of continuing smokers, after both Spanish tobacco control legislations. Thereby, our results could be backing the hypothesis of a switch of smokers to cheaper tobacco products, such as RYO cigarettes $(14,15)$, because the tobacco control policies, particularly increasing of prices, are traditionally focused on conventional cigarettes. Regarding Spain, in recent years, the prices of these products have been surprisingly different, with RYO costing remarkably less than manufactured cigarettes. Moreover, the economic crisis that took place in Spain in 2008 could have affected the shift on tobacco products as has been also reported (10). Bearing this in mind, there is a need to equalize the prices of all tobacco products by applying the same taxing level as, indeed, recommended by the article 6 of the FCTC (39). Furthermore, the highest value of salivary cotinine after Spanish smoking bans, according to the kind of tobacco smoked, was observed in smokers who switched from conventional to RYO cigarettes or to mixed use, and may counteract the popular belief that RYO cigarettes are less harmful than conventional cigarettes (40). This aspect may also be involved in the observed increase in salivary cotinine after the implementation of Spanish smoking bans. Given that many smokers 
switched from conventional cigarettes to other tobacco products, there is a need to implement new instruments to measure dependence not limited to a specific tobacco product.

Finally, $68 \%$ of continuing smokers were on the preparation stage, indicating that they were planning to quit within the next 30 days and that had attempted to quit for at least 24 hours in the past year; also $45.7 \%$ were classified as having low (FTCD score $\leq 4$ ) nicotine dependence. However, according to the Tobacco Control Scale report of $2013(36,37)$, the Spanish score for policies related to treatment to help smokers quit was low (6 out of 10 points) in comparison with other policies. This could mean that smokers may not receive the help needed to succeed when trying to stop smoking or that they are not properly identified. Therefore, there is a need to implement better treatment policies to help smokers stop smoking.

The main limitation of our study is the potential participation bias due to the attrition of the cohort of participants. In this sense, there were statistically significant differences according to age, level of education, and smoking status between the follow-up sample and the participants lost in the follow-up (41). The followed-up sample overestimated young people and smokers in comparison with lost participants. For this reason, the increase in salivary cotinine could be smaller among lost participants. On the other hand, our sample, being a cohort, overestimated the older people compared with the distribution of population in Barcelona. However, we weighted the sample to minimize these limitations and to generate estimations representative of the general population. Moreover, the baseline sample size was representative of the city of Barcelona $(24,25)$ and the longitudinal design maximizes the internal validity of the study. Other potential limitations are those related to potential information biases derived from the use of a self-reported questionnaire to collect information, and non-response. However, trained interviewers administered a face-to-face questionnaire and used the same definition of smoking status in both studies, potentially increasing the internal validity of our results. In addition, we used salivary cotinine, a specific biomarker of nicotine. 
In conclusion, this study shows a significant increase in salivary cotinine concentration among adult continuing smokers after the implementation of both Spanish legislations. Moreover, we observed a shift in the type of tobacco product used, particularly from conventional cigarettes to RYO. Our results, therefore, suggest the need to extend tobacco control policies, focusing on reduction of use of any kind of tobacco product (i.e. equalizing the prices of all tobacco products) and implementing better treatment policies to help smokers stop smoking. 


\section{REFERENCES}

(1) WHO. WHO report on the global tobacco epidemic, 2017 Monitoring tobacco use and prevention policies. Available at:

http://apps.who.int/iris/bitstream/10665/255874/1/9789241512824-eng.pdf?ua=1\&ua=1 (last accessed 28/09/2017)

(2) Tan CE, Glantz SA. Association between smoke-free legislation and hospitalizations for cardiac, cerebrovascular, and respiratory diseases: a meta-analysis. Circulation 2012; 126:2177-2183.

(3) WHO. Prevalence of tobacco smoking 2015; Available at: http://www.who.int/gho/tobacco/use/en/ (last accessed 28/09/2017)

(4) WHO. Global report on trends in tobacco smoking 2000-2025. Available at: http://www.who.int/tobacco/publications/surveillance/reportontrendstobaccosmoking/en/ (last accessed 28/09/2017)

(5) OECD/EU (2016). "Smoking among adults", in Health at a Glance: Europe 2016: State of Health in the EU Cycle, OECD Publishing, Paris. Available at: http://www.oecdilibrary.org/docserver/download/8116231ec022.pdf?expires=1506591197\&id=id\&accname=g uest\&checksum=026132E803EB7D1C3B18C7D7F27145EA (last accessed 28/09/2017)

(6) Gutiérrez-Abejón E, Rejas-Gutiérrez J, Criado-Espegel P, Campo-Ortega EP, Brenas-Villalón MT, Martín-Sobrino N. Smoking impact on mortality in Spain in 2012. Med Clin (Barc) 2015; $145: 520-525$

(7) WHO. The WHO Framework Convention on Tobacco Control: an overview. 2015; Available at: http://www.who.int/fctc/WHO_FCTC_summary_January2015.pdf?ua=1 (last accessed 28/09/2017) 
(8) Fernández E. Spain: going smoke free. Tob Control 2006; 15:79-80.

(9) Fernández E, Nebot M. Spain: Beyond the 'Spanish model' to a total ban. Tob Control 2011; 20:6-7.

(10) Grupo de Trabajo sobre Tabaquismo de la Sociedad Española de Epidemiología.

Evaluación del impacto de la Ley de medidas sanitarias frente al tabaquismo. Madrid:

Ministerio de Sanidad y Política Social. 2009; Available at:

http://www.seepidemiologia.es/monografia.pdf (last accessed 28/09/2017)

(11) Nebot M, López MJ, Ariza C, Pérez-Ríos M, Fu M, Schiaffino A, et al. Impact of the Spanish smoking law on exposure to secondhand smoke in offices and hospitality venues: before-andafter study. Environ Health Perspect 2009; 117:344-347.

(12) Fernández E, Fu M, Pascual JA, López MJ, Pérez-Ríos M, Schiaffino A, et al. Impact of the Spanish smoking law on exposure to second-hand smoke and respiratory health in hospitality workers: a cohort study. PLoS One 2009; 4:e4244.

(13) Hoffman SJ, Tan C. Overview of systematic reviews on the health-related effects of government tobacco control policies. BMC Public Health 2015; 15:744.

(14) Lidón-Moyano C, Martín-Sánchez JC, Saliba P, Graffelman J, Martínez-Sánchez JM. Correlation between tobacco control policies, consumption of rolled tobacco and e-cigarettes, and intention to quit conventional tobacco, in Europe. Tob Control 2017; 26:149-152.

(15) Sureda X, Fu M, Martínez-Sánchez JM, Martínez C, Ballbé M, Pérez-Ortuño R, et al. Manufactured and roll-your-own cigarettes: A changing pattern of smoking in Barcelona, Spain. Environ Res 2017; 155:167-174.

(16) López-Nicolás Á, Cobacho MB, Fernández E. The Spanish tobacco tax loopholes and their consequences. Tob Control 2013; 22:e21-4. 
(17) Rosenberry RZ, Strasser AA, Canlas LL, Potts JL, and Pickworth WB. Make Your Own Cigarettes: Characteristics of the Product and the Consumer. Nicotine Tob Res 2013; 15:14531457.

(18) Nuca C, Amariei C, Badea V, Zaharia A, Bucur L, Arendt C. Salivary cotinine - Biomarker of tobacco consumption in the assessment of passive smoking prevalence. Farmacia 2012; 60:662-674.

(19) Martínez-Sánchez JM, Fernández E, Fu M, Pérez-Ríos M, López MJ, Ariza C et al. Impact of the Spanish smoking law in smoker hospitality workers. Nicotine Tob Res 2009: 11:1099-1106.

(20) Jarvis M, Tunstall-Pedoe H, Feyerabend C, Vesey C, Salloojee Y. Biochemical markers of smoke absorption and self reported exposure to passive smoking. J Epidemiol Community Health 1984; 38:335-339.

(21) J. L. Repase, J. Jinot, S. Bayard, K. Emmons, K. Hammond. Air nicotine and saliva cotinine as indicators of workplace passive smoking exposure and risk. Risk analysis 1998; 18:71-83.

(22) Benowitz NL. Biomarkers of Cigarette Smoking. Measurement in the Analysis and Treatment of Smoking Behavior: National Institute on Drug Abuse; 1983. p. 93-11.

(23) Fu M, Fernández E, Pascual JA, Martínez-Sánchez JM, Agudo A, Montcada A et al. Stages of change, smoking characteristics, and cotinine concentrations in smokers: Setting priorities for smoking cessation. Prev Med 2011; 139-145.

(24) Fu M, Fernández E, Martínez-Sánchez JM, Pascual JA, Schiaffino A, Agudo A, et al. Salivary cotinine concentrations in daily smokers in Barcelona, Spain: a cross-sectional study. BMC Public Health 2009; 9:320. 
(25) Martínez-Sánchez JM, Fernández E, Fu M, Pascual JA, Ariza C, Agudo A, et al. Assessment of exposure to secondhand smoke by questionnaire and salivary cotinine in the general population of Barcelona, Spain (2004-2005). Prev Med 2009; 48:218-223.

(26) Pérez-Ortuño R, Martínez-Sánchez JM, Fernández E, Pascual JA. High-throughput wide dynamic range procedure for the simultaneous quantification of nicotine and cotinine in multiple biological matrices using hydrophilic interaction liquid chromatography-tandem mass spectrometry. Anal Bioanal Chem 2015; 407:8463-8473.

(27) Blackford AL, Yang G, Hernández-Avila M, Przewozniak K, Zatonski W, Figueiredo V, et al. Cotinine concentration in smokers from different countries: relationship with amount smoked and cigarette type. Cancer Epidemiol Biomarkers Prev 2006; 15:1799-1804.

(28) DiClemente CC, Prochaska JO, Fairhurst SK, Velicer WF, Velasquez MM, Rossi JS. The process of smoking cessation: an analysis of precontemplation, contemplation, and preparation stages of change. J Consult Clin Psychol 1991; 59:295-304.

(29) Fagerström K. Determinants of tobacco use and renaming the FTND to the Fagerstrom Test for Cigarette Dependence. Nicotine Tob Res 2012; 14:75-8.

(30) Hughes JR. The hardening hypothesis: is the ability to quit decreasing due to increasing nicotine dependence? A review and commentary. Drug Alcohol Depend 2011; 117:111-117. (31) Gallus S, Pacifici R, Colombo P, La Vecchia C, Garattini S, Apolone G, et al. Tobacco dependence in the general population in Italy. Ann Oncol 2005; 16:703-706.

(32) Lund M, Lund KE, Kvaavik E. Hardcore smokers in Norway 1996-2009. Nicotine Tob Res 2011; 13:1132-1139. 
(33) Fernández E, Lugo A, Clancy L, Matsuo K, La Vecchia C, Gallus S. Smoking dependence in 18 European countries: Hard to maintain the hardening hypothesis. Prev Med 2015; 81:314319.

(34) Fu M, Martínez-Sánchez JM, Agudo A, Pascual JA, Ariza C, Moncada A, et al. Nicotine depedence and salivary cotinine concentration in daily smokers. Eur J Cancer Prev 2012; 21:96102.

(35) Etter J, Vu Duc T, Perneger TV. Validity of the Fagerström test for nicotine dependence and of the Heaviness of Smoking Index among relatively light smokers. Addiction 1999; 94:269281.

(36) Joossens L, Raw M. The Tobacco Control Scale 2013 in Europe. Association of European Cancer Leagues (ECL) 2014.

(37) Grupo de Trabajo sobre Tabaquismo de la Sociedad Española de Epidemiología.

Evaluación de las políticas de control del tabaquismo en España (Leyes 28/2005 y 42/2010). Revisión de la evidencia. 2017; Available at:

http://www.seepidemiologia.es/documents/dummy/V9.0\%20\%20Libro\%20Tabaquismo\%202017\%20-\%20Abierto\%20Final.pdf (last accessed 28/09/2017) (38) Lidón-Moyano C, Fu M, Ballbè M , Martín-Sánchez JC, Matilla-Santander N, Martínez C, et al. Impact of the Spanish smoking laws on tobacco consumption and secondhand smoke exposure: A longitudinal population study. Addict Behav 2017; 75:30-35.

(38) WHO. WHO framework convention on tobacco control. Available at: http://whqlibdoc.who.int/publications/2003/9241591013.pdf?ua=1 (last accessed 28/09/2017) 
(39) O'Connor RJ, McNeill A, Borland R, Hammond D, King B, Boudreau C, et al. Smokers'

beliefs about the relative safety of other tobacco products: findings from the ITC collaboration. Nicotine Tob Res 2007; 9:1033-1042.

(40) Lidón-Moyano C, Martínez-Sánchez JM, Fu M, Ballbè M, Martín-Sánchez JC, Martínez C, et al. Impact of the Spanish smoking legislationsin the adoption of smoke-free rules at home: a longitudinal study in Barcelona (Spain). Tob.Control 2016; 26:557-56 
Figure1. Flow chart of sample followed-up from Barcelona, Spain, in 2013-2014.

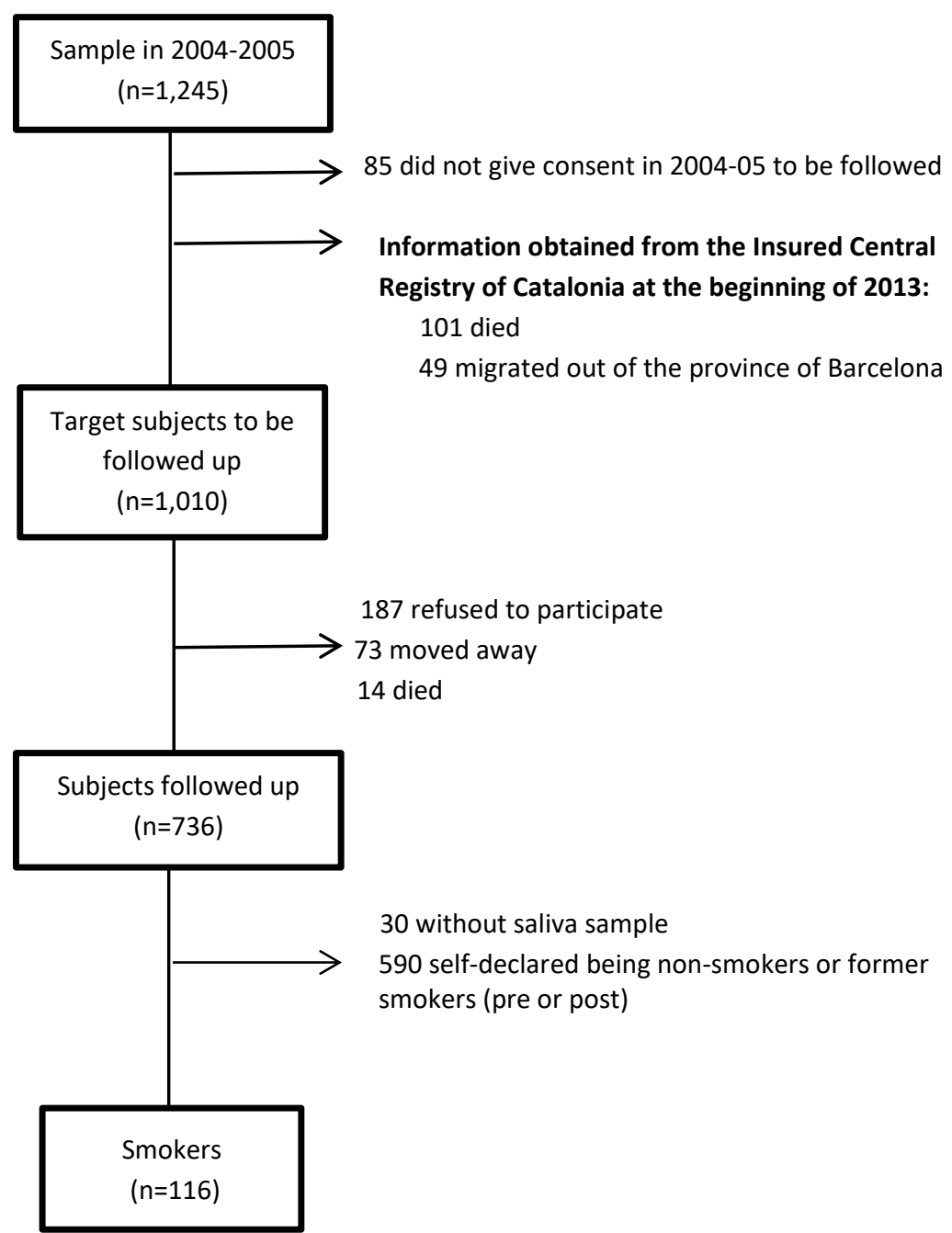

Pre: before Spanish smoke-free bans (2004-2005).

Post: after Spanish smoke-free bans (2013-2014). 
Table 1: Differences between follow-up sample and lost in both stages of the follow-up (prevalence for qualitative variables and mean (standard deviation) for quantitative variable).

\begin{tabular}{|c|c|c|c|}
\hline & $\begin{array}{c}\text { Lost } \\
\text { (both stages) } \\
n=509\end{array}$ & \begin{tabular}{|c|} 
Follow-up \\
$n=736$
\end{tabular} & P-value \\
\hline Sex & & & \\
\hline men & $42.2 \%$ & $45.7 \%$ & $0.257^{\mathrm{a}}$ \\
\hline women & $57.8 \%$ & $54.3 \%$ & \\
\hline Age (years) & $58(39)$ & $49(26.25)$ & $<0.001^{b}$ \\
\hline Age & & & \\
\hline$<45$ & $35.2 \%$ & $43.9 \%$ & $<0.001^{a}$ \\
\hline $45-64$ & $22 \%$ & $37.5 \%$ & \\
\hline$>=65$ & $42.8 \%$ & 18.65 & \\
\hline Educational level & & & \\
\hline Low & $53 \%$ & $38.9 \%$ & $<0.001^{\mathrm{a}}$ \\
\hline Intermediate & $20.5 \%$ & $24.2 \%$ & \\
\hline High & $26.5 \%$ & $36.9 \%$ & \\
\hline Smoking status (follow-up) & & & \\
\hline smoker & $24.1 \%$ & $31.1 \%$ & $0.009^{a}$ \\
\hline no smoker & $75.9 \%$ & $68.9 \%$ & \\
\hline
\end{tabular}

a. Chi-square test

b. Mann-Whitney test 
Figure 2. Changes in salivary cotinine concentration (log scale), in the number of cigarettes smoked daily (log scale), and in the Fagerström Test for Cigarette Dependence (before and after the implementation of both Spanish tobacco control legislations).

B

Log scale salivary cotinine (ng/ml)
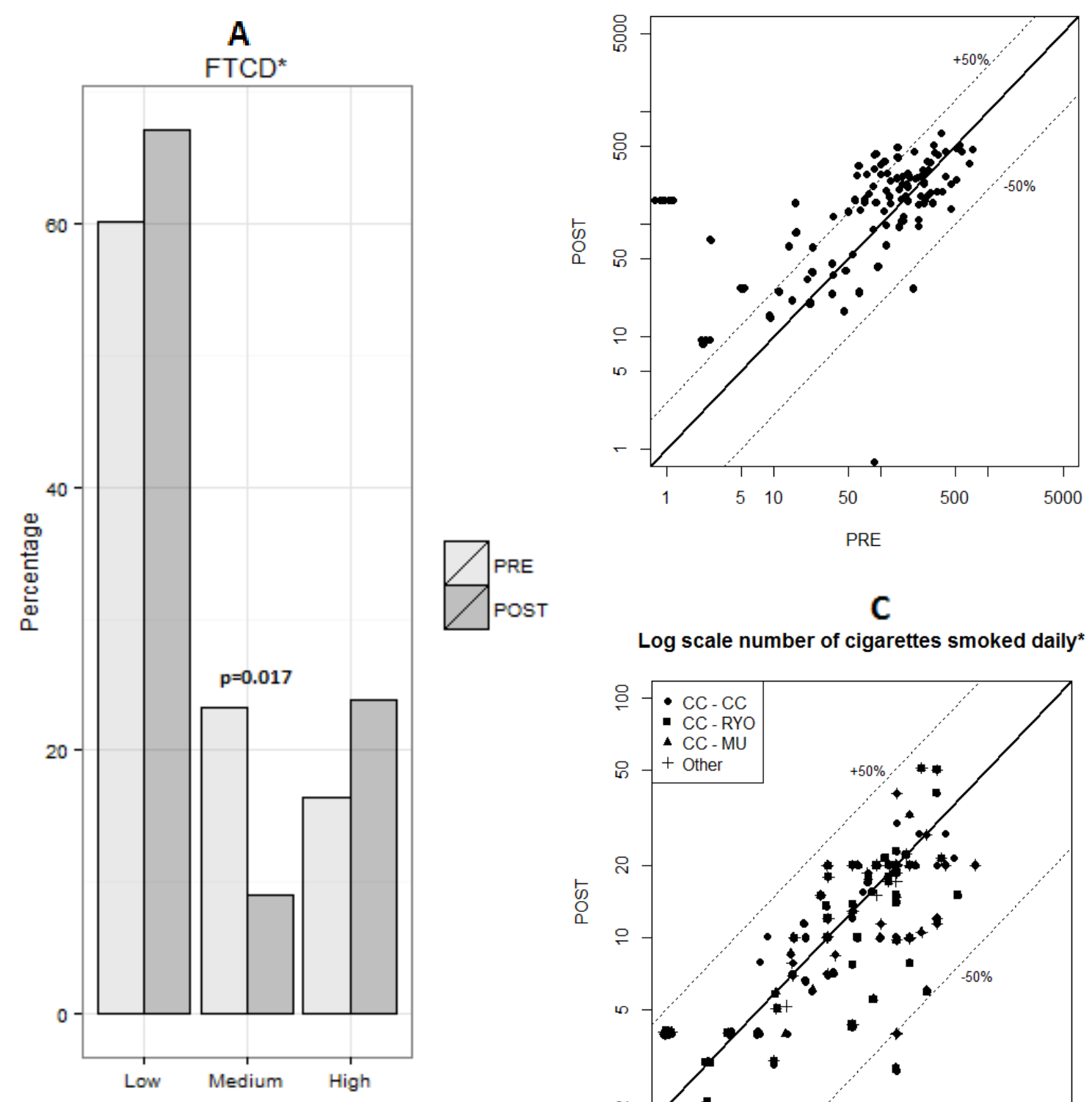

C

Log scale number of cigarettes smoked daily*

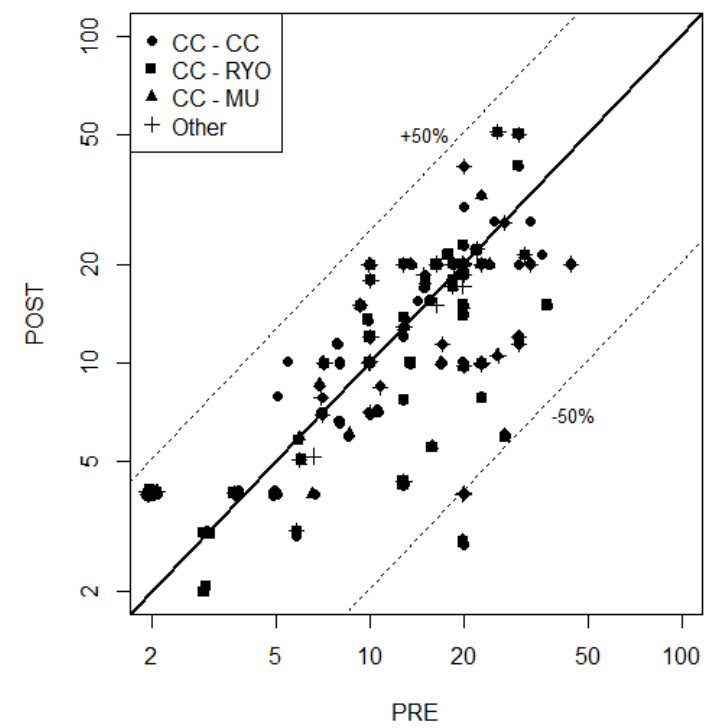

*Daily smokers

FTCD categorized as 'low' ( $\leq 4)$, 'medium' (5) and 'high' ( $\geq 6$ )

Statistically significant differences were only found in the 'medium' group when comparing FTCD categories pre and post legislation (proportion test).

Pre: before Spanish smoke-free bans (2004-2005).

Post: after Spanish smoke-free bans (2013-2014). 
Table 2. Geometric mean (GM) and geometric standard deviation (GSD), adjusted linear mixed effect coefficient (\% change*) and their $95 \%$ confidence interval $(95 \% \mathrm{Cls})$ of salivary cotinine concentration $(\mathrm{ng} / \mathrm{mL})$ according to sociodemographic variables, kind of tobacco smoked, Fagerström Test for Cigarette Dependence score (FTCD) and stages of change before (20042005) and after (2013-2014) the implementation of both Spanish tobacco control legislations.

\begin{tabular}{|c|c|c|c|c|c|}
\hline & $n^{a}$ & \begin{tabular}{|c|} 
GM (GSD) \\
ng/mL (PRE)
\end{tabular} & $\begin{array}{c}\mathrm{GM} \text { (GSD) } \\
\mathrm{ng} / \mathrm{mL} \text { (POST) }\end{array}$ & $\%$ change* $(95 \% \mathrm{Cl})$ & p-value \\
\hline Overall & 116 & $91.1(0.16)$ & $117.3(0.18)$ & $28.7(4.9 ; 58.0)$ & 0.015 \\
\hline \multicolumn{6}{|l|}{ Sex } \\
\hline Men & 62 & $103.9(0.26)$ & $135.2(0.28)$ & $30.1(-0.1 ; 69.4)$ & 0.051 \\
\hline Women & 54 & $78.3(0.19)$ & $99.6(0.22)$ & $27.2(-7.8 ; 75.6)$ & 0.143 \\
\hline \multicolumn{6}{|l|}{ Age } \\
\hline $26-44$ & 62 & $104.3(0.15)$ & $174.3(0.09)$ & $67.2(30.7 ; 113.8)$ & $<0.001$ \\
\hline $45-64$ & 34 & $132.7(0.18)$ & $112.9(0.28)$ & $-14.9(-46.5 ; 35.2)$ & 0.494 \\
\hline $65-98$ & 20 & $30.7(0.22)$ & $35.3(0.34)$ & $15.0(-13.5 ; 52.9)$ & 0.336 \\
\hline \multicolumn{6}{|l|}{ Educational level } \\
\hline Low & 46 & $102.1(0.32)$ & $112.5(0.35)$ & $10.2(-11.8 ; 37.7)$ & 0.392 \\
\hline Intermediate & 33 & $100.9(0.22)$ & $142.2(0.27)$ & $40.9(-21.1 ; 151.6)$ & 0.246 \\
\hline High & 37 & $72.4(0.16)$ & $104.0(0.24)$ & $43.8(13.9 ; 81.4)$ & 0.002 \\
\hline \multicolumn{6}{|l|}{ Kind of tobacco smoked $^{1}$} \\
\hline $\mathrm{CC}-\mathrm{CC}$ & 64 & $106.6(0.18)$ & $114.9(0.20)$ & $7.8(-18.0 ; 41.9)$ & 0.590 \\
\hline $\mathrm{CC}-\mathrm{RYO}$ & 14 & $145.6(0.16)$ & $243.2(0.12)$ & $70.0(16.7 ; 138.8)$ & 0.005 \\
\hline $\mathrm{CC}-\mathrm{MU}$ & 9 & $205.2(0.16)$ & $236.9(0.14)$ & $15.5(-12.1 ; 51.6)$ & 0.301 \\
\hline Other & 21 & $74.3(0.55)$ & $80.8(0.63)$ & $8.8(-15.2 ; 39.5)$ & 0.509 \\
\hline \multicolumn{6}{|l|}{ FTCD $_{d}$ score (PRE) } \\
\hline Low & 53 & $115.7(0.12)$ & $137.4(0.18)$ & $18.7(-15.3 ; 66.3)$ & 0.319 \\
\hline Medium & 20 & $86.6(0.43)$ & $103.3(0.39)$ & $19.3(-0.9 ; 43.5)$ & 0.062 \\
\hline High & 14 & $237.6(0.15)$ & $272.3(0.12)$ & $14.6(-12.8 ; 50.7)$ & 0.329 \\
\hline \multicolumn{6}{|l|}{ Stages of Change (PRE) } \\
\hline Precontemplation & 6 & $186.7(0.36)$ & $257.1(0.20)$ & $37.7(-26.0 ; 156.3)$ & 0.313 \\
\hline Contemplation & 9 & $136.8(0.30)$ & $172.5(0.25)$ & $26.1(-11.5 ; 79.8)$ & 0.200 \\
\hline Preparation & 79 & $93.5(0.21)$ & $115.1(0.23)$ & $23.1(-7.3 ; 63.6)$ & 0.151 \\
\hline
\end{tabular}

FTCD : FTCD daily smokers.

$\mathrm{n}^{\mathrm{a}}$ : The sum does not up the total for some variables because of some missing values.

Pre: Before Spanish smoke-free bans (2004-2005).

Post: After Spanish smoke-free bans (2013-2014).

$\%$ change*: Adjusted by sex, age, educational level.

P-value: Obtained through adjusted linear mixed effect coefficient.

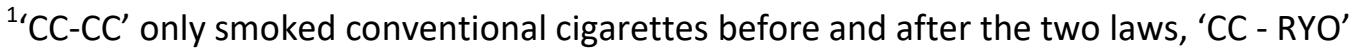
switched from conventional to RYO cigarettes, ' $\mathrm{CC}-\mathrm{MU}$ ' switched from conventional to mixed use (CC and RYO) and 'Other' indicates other possible options. 
Table 3. Prevalence and generalized linear mixed effect coefficient (Prevalence Ratio PR), or mean (standard deviation) and mean difference, smoking characteristics before (2004-2005) and after (2013-2014) the implementation of both Spanish tobacco control legislations.

\begin{tabular}{|c|c|c|c|c|c|}
\hline & $n^{a}$ & PRE & POST & PR $(95 \% \mathrm{Cl})$ & p-value \\
\hline \multicolumn{6}{|l|}{ Type of cigarettes (\%) } \\
\hline Regular & 66 & 66.6 & 76.3 & $1.15(1.02,1.28)$ & $0.019^{1}$ \\
\hline Other & 33 & 33.4 & 23.7 & $0.71(0.50,0.99)$ & $0.049^{1}$ \\
\hline \multicolumn{6}{|l|}{ Type of tobacco (\%) } \\
\hline Blond & 82 & 82.2 & 88.1 & $1.07(0.99,1.16)$ & $0.097^{1}$ \\
\hline Black & 18 & 17.8 & 11.9 & $0.67(0.42,1.06)$ & $0.086^{1}$ \\
\hline \multicolumn{6}{|l|}{ Filter (\%) } \\
\hline Yes & 97 & 98 & 98 & $1.00(0.98,1.02)$ & $0.96^{1}$ \\
\hline No & 2 & 2 & 2 & $1.04(0.35,3.07)$ & $0.94^{1}$ \\
\hline $\begin{array}{l}\text { Length of cigarettes left after } \\
\text { smoking in } \mathrm{cm} \text { (mean, SD) }\end{array}$ & 107 & $1.13(0.12)$ & $2.26(0.39)$ & - & $0.023^{2}$ \\
\hline \multicolumn{6}{|l|}{ Frequency of inhalation (\%) } \\
\hline Continuous & 13 & 11.7 & 16.9 & $1.44(0.83,2.52)$ & $0.198^{1}$ \\
\hline Regular & 65 & 60.1 & 73 & $1.21(0.86,1.71)$ & $0.264^{1}$ \\
\hline Scarce & 30 & 28.2 & 10.1 & $0.36(0.14,0.93)$ & $0.036^{1}$ \\
\hline \multicolumn{6}{|l|}{ Depth of inhalation (\%) } \\
\hline Superficial & 17 & 16.2 & 24.7 & $1.56(0.73,3.32)$ & $0.246^{1}$ \\
\hline Intermediate & 45 & 41.4 & 17.8 & $0.43(0.26,0.72)$ & $0.001^{1}$ \\
\hline Deep & 46 & 42.4 & 57.5 & $1.35(1.13,1.61)$ & $0.001^{1}$ \\
\hline
\end{tabular}

$\mathrm{n}^{\text {a }}$ : The sum does not up the total for some variables because of some missing values.

Pre: Before Spanish smoke-free ban of 2004-2005.

Post: After Spanish smoke-free ban of 2013-2014.

PR: Prevalence ratio obtained through generalized linear mixed effect coefficient.

${ }^{1}$ Generalized linear mixed effect coefficient $p$-value.

${ }^{2} \mathrm{~T}$-test for paired samples. 